\title{
Ocean waves propagating over a porous seabed: Residual and oscillatory mechanisms
}

\author{
JENG DongSheng ${ }^{1,3}$, SEYMOUR Brian ${ }^{2}$, GAO FuPing ${ }^{3 \dagger}$ \& WU YingXiang ${ }^{3}$ \\ ${ }^{1}$ School of Civil Engineering, The University of Sydney, Sydney, NSW 2006, Australia; \\ ${ }^{2}$ Department of Mathematics, The University of British Columbia, Vancouver, V6Z 1Z2, Canada; \\ ${ }^{3}$ Institute of Mechanics, Chinese Academy of Sciences, Beijing 100080, China
}

Two mechanisms for the wave-induced pore pressures in a porous seabed, i.e. oscillatory and residual excess pore pressures, have been observed in laboratory experiments and field measurements. Most previous investigations have focused on one of the mechanisms individually. In this paper, an analytical solution for the wave-induced residual pore pressure, which is not available yet, is derived, and compared with the existing experimental data. With the new solution, a parametric analysis is performed to clarify the applicable ranges of two mechanisms. Then, a simplified approximation for the prediction of wave-induced liquefaction potential is proposed for engineering practice.

seabed, pore pressure, residual, oscillatory, wave loading

One of the important marine geotechnical engineering considerations for many engineering installations in oceanic environments, such as platform, pipeline and anchors, is the liquefaction potential of seabed due to ocean waves. Numerous examples of liquefaction have been reported in the literature, such as, floatation of pipeline during storm ${ }^{[1]}$, sinking of several measuring instructions in Mississippi Delta ${ }^{[2]}$ and subsidence of offshore breakwaters at Niagata Coast, Japan ${ }^{[3]}$.

Two significant mechanisms accounting for wave-induced soil response, including oscillatory pore pressure and residual pore pressure, have been observed in laboratory experiments and field measurements ${ }^{[4]}$, as shown in Figure 1. The first mechanism, termed as transient or oscillatory excess pore pressure, is accompanied by the attenuation of amplitude and the phase lag in pore pressure changes ${ }^{[5,6]}$. This mechanism is more important for unsaturated marine sediments and deeper water region. The second mechanism, termed as the residual pore pressure, is the build-up of excess pore pressure caused by contraction of the soil under the action of cyclic loading ${ }^{[7,8]}$. This mechanism is similar to the earthquake-induced pore pressure accumulation.

Numerous investigations for ocean waves propagating over a porous seabed have been carried

Received January 7, 2005; accepted July 28, 2006 doi: 10.1007/s11431-007-2018-5

${ }^{\dagger}$ Corresponding author (email: fpgao@imech.ac.cn)

Supported by the National Natural Science Foundation of China (Grant Nos. 50509022 and 10532070) and the 'Tenth Five-year Plan' of the Chinese Academy of Sciences (Grant No. KJCX2-SW-L03) 
out since the $1970 \mathrm{~s}^{[5-10]}$. Among these, both mechanisms have been considered individually. The applicable range of each mechanism is unclear, due to no analytical solutions for residual pore pressure being available. Thus, in this paper, we derive a new analytical approximation for the pore pressure build-up due to waves through a Laplace's transformation. A comparison with the existing experimental data will be presented for the verification of the model. Then we further clarify the applicable range of two mechanisms. Finally, a simplified approximation for the prediction of liquefaction potential will be proposed for engineering practice.

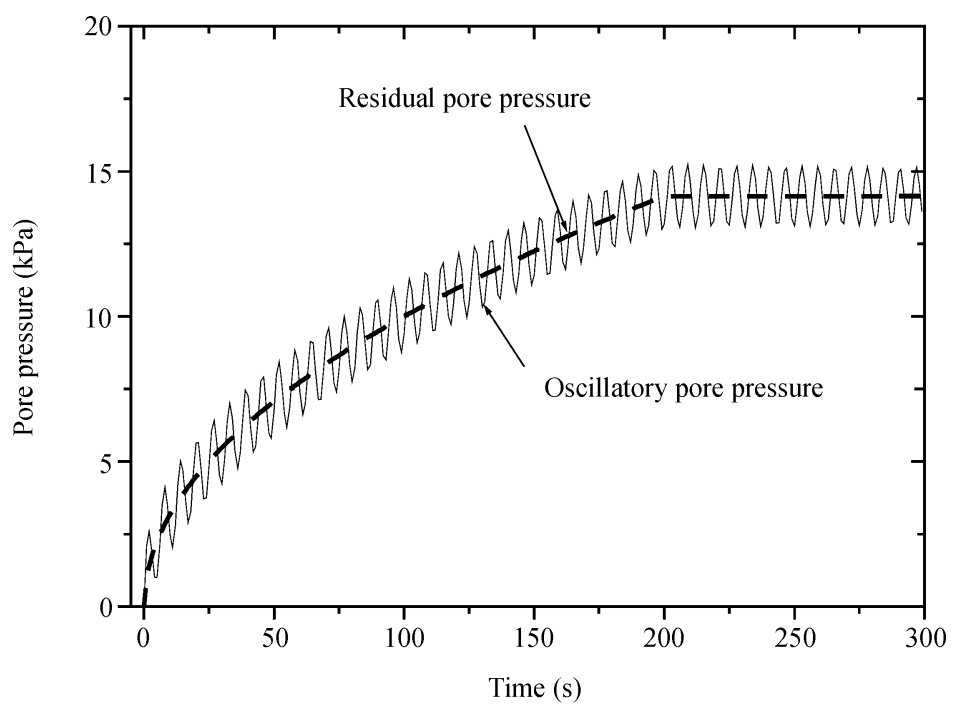

Figure 1 Illustration of wave-induced pore pressure in seabed (not in scale).

\section{Theoretical formulation}

Considering the problem of wave-seabed interaction, as shown in Figure 2, the wave-induced pore pressure in a porous seabed $(p)$ can be written as

$$
p(x, z, t)=\tilde{p}(x, z, t)+\bar{p}(z, t),
$$

where $\tilde{p}$ represents the oscillatory pore pressure, while $\bar{p}$ represents the period-averaged pore pressure, which is defined by

$$
\bar{p}=\frac{1}{T} \int_{t}^{t+T} p d t .
$$

In (1), in order to simplify the problem, we decouple the oscillatory and residual mechanisms as the first approximation. This is because no model for coupling two mechanisms has been available yet. To date, there is no model for the determination the oscillating shear stress at the instant during cyclic loading $^{[11]}$. Therefore, we decouple two mechanisms in this study.

It is noted that the oscillatory pore pressure $(\tilde{p}(x, z, t))$ fluctuates in both spatial and temporal domains while wave is propagating over the porous seabed. However, the residual pore pressure $(\bar{p})$ is built up from the accumulation of pore pressure at a particular location during the wave loading. Thus, the residual pore pressure can be treated as one-dimensional problem, i.e., $\bar{p} \equiv \bar{p}(z, t)$ in $(1)$. 


\subsection{Oscillatory pore pressure}

To derive the analytical solution, the following assumptions have been made:

- The seabed is porous and horizontal.

- The pore fluid and soil are compressible.

- The soil matrix obeys the Hooke's law.

- Linear wave loading is the only external loading.

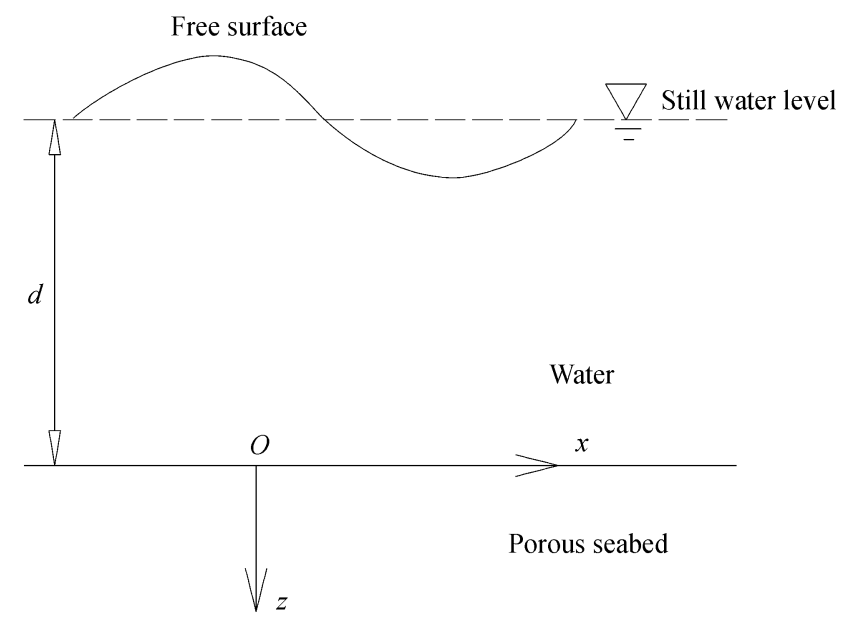

Figure 2 Sketch of ocean waves propagating over a porous seabed.

In this study, we ignore the viscous effect, i.e., the wave boundary layer and bottom shear stresses along the seabed surface are ignored. It is valid for most cases. In fact, the inclusion of wave-induced bottom shear stress does not play a significant role in the evaluation of the wave-induced pore pressure within marine sediments ${ }^{[6]}$.

Based on Biot's consolidation equation ${ }^{[9]}$, the oscillatory pore pressure can be solved with the following boundary value problem, as presented in Jeng ${ }^{[6]}$ :

$$
\begin{aligned}
& K \nabla^{2} \tilde{p}=n_{e} \beta \gamma_{w} \frac{\partial \tilde{p}}{\partial t}+\gamma_{w} \frac{\partial \xi}{\partial t}, \\
& G \nabla^{2} u+\frac{G}{1-2 \mu} \frac{\partial \xi}{\partial x}=\frac{\partial \tilde{p}}{\partial x}, \\
& G \nabla^{2} w+\frac{G}{1-2 \mu} \frac{\partial \xi}{\partial z}=\frac{\partial \tilde{p}}{\partial z},
\end{aligned}
$$

where $u$ and $w$ are the soil displacements in the $x$ - and $z$-directions, respectively, $K$ is soil permeability, $n_{e}$ is soil porosity, $\gamma_{w}$ is the unit weight of pore fluid, $G$ is shear modulus of soil, $\mu$ is Poisson's ratio, and the volumetric strain $(\xi)$ and compressibility of pore fluid $(\beta)$ are defined by

$$
\xi=\frac{\partial u}{\partial x}+\frac{\partial w}{\partial z} \text { and } \beta=\frac{1}{K^{\prime}}+\frac{1-S}{P_{w o}},
$$

in which $K^{\prime}$ is the true bulk modulus of elasticity of water, $S$ is the degree of saturation, and $P_{w o}$ is the absolute static water pressure.

The stress-strain relationship is given by 


$$
\begin{gathered}
\sigma_{x}^{\prime}=2 G\left(\frac{\partial u}{\partial x}+\frac{\mu \xi}{1-2 \mu}\right) \\
\sigma_{z}^{\prime}=2 G\left(\frac{\partial w}{\partial z}+\frac{\mu \xi}{1-2 \mu}\right), \\
\tau=G\left(\frac{\partial u}{\partial z}+\frac{\partial w}{\partial x}\right)
\end{gathered}
$$

where $\sigma_{x}^{\prime}$ and $\sigma_{z}^{\prime}$ are the effective normal stresses in the $x$ - and $z$-directions, respectively, and $\tau$ is the shear stress.

The above governing equations $(3 a)-(3 c)$ can be solved with appropriate boundary conditions. First, the pore pressure is identical to the dynamic wave pressure on the seabed surface, while vertical effective normal stress vanishes. Also, the shear stress will become zero on the seabed surface, because of neglecting the wave boundary layer near the seabed surface.

$$
\begin{aligned}
& \tilde{p}(x, 0, t)=\frac{\gamma_{w} H}{2 \cosh k d} \cos (k x+\omega t)=P_{b} \cos (k x+\omega t), \\
& \sigma_{z}^{\prime}(x, 0, t)=\tau(x, 0, t)=0,
\end{aligned}
$$

where $H$ is wave height, $d$ is water depth, $k$ is wave number, $\omega(=2 \pi / T, T$ is wave period $)$ is wave frequency, and $P_{b}$ is the amplitude of dynamic wave pressure upon seabed surface. Second, the soil displacements and pore pressure will vanish at the impermeable seabed bottom $(z \rightarrow \infty)$, i.e.

$$
u(x, \infty, t)=w(x, \infty, t)=p(x, \infty, t)=0 .
$$

The analytical solution to the wave-induced oscillatory soil response in a saturated seabed was derived by Jeng and $\mathrm{Hsu}^{[10]}$. For a saturated seabed of infinite thickness, the pore pressure $(\tilde{p})$ and shear stress $(\tau)$ can be expressed as

$$
\begin{aligned}
& \tilde{p}=P_{b} \exp (-k z) \cos (k x+\omega t), \\
& \tau=P_{b} k z \exp (-k z) \cos (k x+\omega t) .
\end{aligned}
$$

\subsection{Residual pore pressure}

The residual pore pressure $(\bar{p})$ in homogenous, isotropic soil can be derived from the one-dimensional Biot's consolidation equation as

$$
\frac{\partial \bar{p}}{\partial t}=c_{v} \frac{\partial^{2} \bar{p}}{\partial z^{2}}+f(z)
$$

in which $c_{v}$ is the coefficient of consolidation, given by

$$
c_{v}=\frac{2 G K(1-\mu)}{\gamma_{w}(1-2 \mu)}
$$

and the source term $f(z)$ is defined by ${ }^{[11]}$

$$
\begin{gathered}
f(z)=A z \exp (-\lambda z), \\
A=\frac{\gamma^{\prime}\left(1+2 K_{o}\right)}{3 T}\left(\frac{3 P_{b} k}{\alpha\left(1+2 K_{o}\right) \gamma^{\prime}}\right)^{1 / \eta},
\end{gathered}
$$




$$
\lambda=\frac{k}{\eta} \quad \text { and } \quad \gamma^{\prime}=\gamma_{s}-\gamma_{w}
$$

in which $\gamma_{s}$ is the unit weight of soil, $K_{o}$ is the lateral earth pressure coefficient in rest state, and $\alpha$ and $\eta$ are two empirical constants, which are functions of the relative density of soil $\left(D_{r}\right)^{[12]}$.

Boundary and initial conditions for the above governing equations are

$$
\bar{p}(0, t)=\bar{p}(z, 0)=0 \text {, and } \bar{p}(\infty, t)=0 .
$$

The above boundary value problem can be solved by Laplace's transformation. The solution to the wave-induced residual pore pressure can be written as

$$
\bar{p}=\frac{2 A}{c_{v} \lambda^{3}}\left[1-\left(\frac{\lambda z}{2}+1\right) \exp (-\lambda z)-\frac{1}{\pi} \int_{0}^{\infty} \frac{\exp \left(-r c_{v} \lambda^{2} t\right)}{r(r+1)^{2}} \sin (\sqrt{r} \lambda z) d r\right] .
$$

Note that the integral term in (13) will vanish for large time $(t)$. The detailed derivation of (13) can be found in Appendix A.

A comparison of the present model with the existing experimental data ${ }^{[13]}$ is illustrated in Figure 3. The equilibrium residual pore pressure $\left(P_{\text {res }}\right)$ is defined by $(14 \mathrm{~b})$. And the present analytical solution overall agrees with the experimental data. The difference between the analytical solution and experimental data may come from the seabed thickness, as the experiments were conducted for the case of finite thickness.
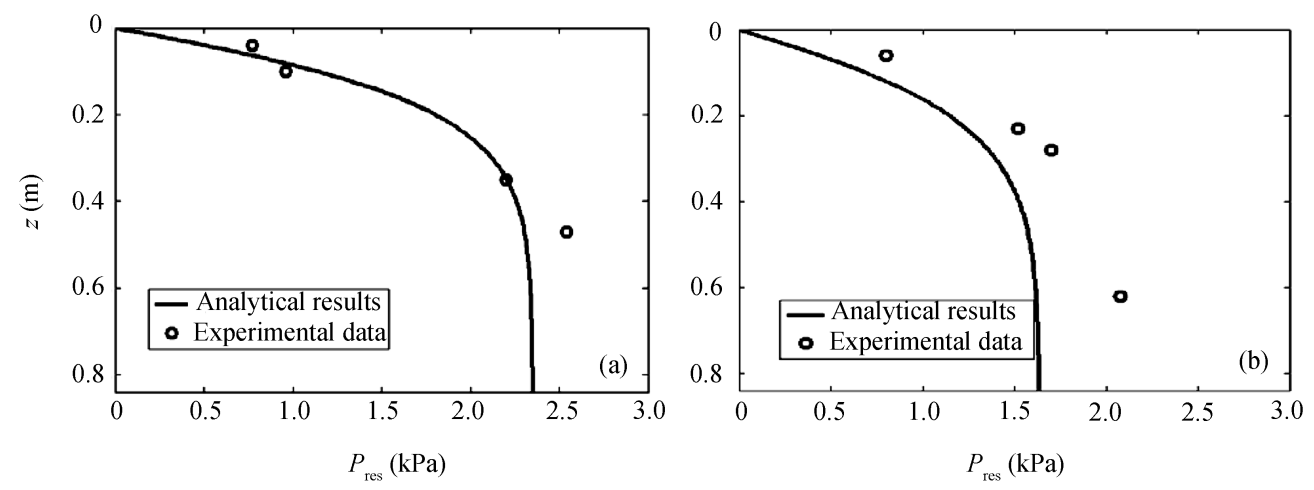

Figure 3 Comparison of the analytical solution with experimental data ${ }^{[13]}$ (Input data are tabulated in Table 1).

\section{Parametric analyses}

The first objective of this study is to clarify the applicable range of two mechanisms. Now, we compare the amplitude of the oscillatory pore pressure $(|\tilde{p}|)$ and equilibrium residual pore pressure $(\bar{p}(z, \infty))$, which are

$$
\begin{aligned}
& P_{\text {osc }}=|\tilde{p}|=P_{b} \exp (-k z), \\
& P_{\text {res }}=\bar{p}(z, \infty)=\frac{2 A}{c_{v} \lambda^{3}}\left[1-\left(\frac{\lambda z}{2}+1\right) \exp (-\lambda z)\right] .
\end{aligned}
$$

To examine the role of each mechanism, we define the amplitude ratio $(\varepsilon)$

$$
\varepsilon=\frac{P_{\text {res }}}{P_{\text {osc }}}
$$


Figure 4 illustrates the results of (15) for various values of $\varepsilon$ with $T=10 \mathrm{~s}$. The lines represent the critical relationship between $H / L$ and $d / L$. The region below the curve which denotes transient (oscillating) mechanism dominates the magnitude of pore pressure; while the region above the curve which denotes residual mechanism dominates the pore pressure. The critical curve will move up as the amplitude ratio $(\varepsilon)$ increases. The choice of $\varepsilon$ depends on the requirement of the design.

Table 1 Input data for the comparisons in Figure 3

\begin{tabular}{lcc}
\hline & Wave characteristics & \\
\hline & & $(\mathrm{a})$ \\
Wave period $(T)$ & $1.76 \mathrm{~s})$ & $2.02 \mathrm{~s}$ \\
Water depth $(d)$ & $0.5 \mathrm{~m}$ & $0.5 \mathrm{~m}$ \\
Wave length $(L)$ & $3.473 \mathrm{~m}$ & $4.103 \mathrm{~m}$ \\
Wave height $(H)$ & $0.22 \mathrm{~m}$ & $0.21 \mathrm{~m}$ \\
\hline & Soil characteristics & $0.84 \mathrm{~m}$ \\
Seabed thickness $(h)$ & 0.49 & 0.46 \\
Poisson's ratio $(\mu)$ & $5.5 \times 10^{5} \mathrm{~N} / \mathrm{m}^{2}$ \\
Soil porosity $\left(n_{e}\right)$ & $4.0 \times 10^{-8} \mathrm{~m} / \mathrm{s}$ \\
Shear modulus $(G)$ & $18.2 \times 10^{3} \mathrm{~N} / \mathrm{m}^{3}$ \\
Soil permeability $(K)$ & $9.806 \times 10^{3} \mathrm{~N} / \mathrm{m}^{3}$ \\
Unit weight of soil $\left(\gamma_{s}\right)$ & 1.0 \\
Unit weight of pore fluid $\left(\gamma_{w}\right)$ & 0.4 \\
Degree of saturation $(S)$ & $1.165 \times 10^{-4}$ \\
Coefficient of earth pressure $\left(K_{\mathrm{o}}\right)$ & 0.246 \\
Consolidation coefficient $\left(c_{v}\right)$ & 0.165 \\
$\alpha$ & & \\
$\eta$ & & \\
\hline
\end{tabular}

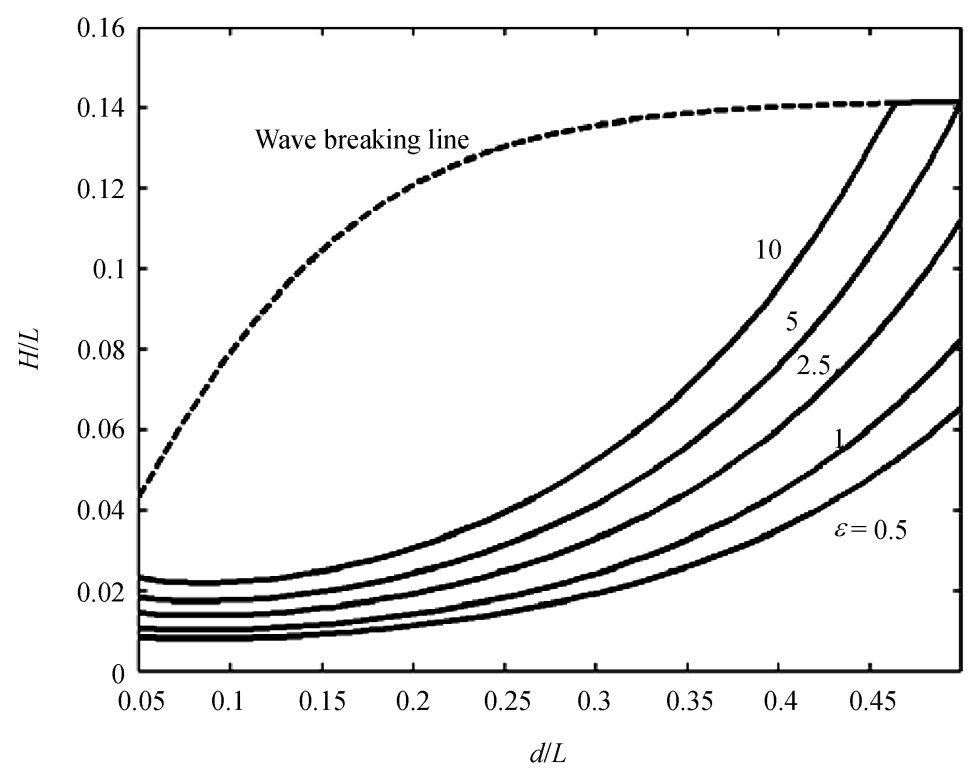

Figure 4 Distribution of critical wave steepness $(H / L)$ versus relative water depth $(d / L)$ for various value of amplitude ratios $(\varepsilon)$. (Input data: $n_{e}=0.33, \mu=0.33, K=10^{-6} \mathrm{~m} / \mathrm{s}, G=5 \times 10^{6} \mathrm{~N} / \mathrm{m}^{2}, \alpha=0.246, \eta=0.25, c_{v}=0.0022$ and $T=10 \mathrm{~s}$ ).

As shown in Figure 4, the residual mechanism is more important for large wave conditions, while oscillatory mechanism dominates the pore pressure under small wave conditions, if we use $\varepsilon=1$ as a critical condition. However, all previous investigations ${ }^{[7,8]}$ concerned with residual 
mechanism have been based on linear wave theory. It is well known that linear wave theory is insufficient from the aspect of ocean engineering, but it can provide some fundamental knowledge for engineers, since it is the leading order solution.

\section{Simplified approximations for engineering practice}

For engineers, the most important task is to examine where liquefaction will occur and how deep it is. The criterion of residual liquefaction is

$$
\frac{P_{\text {res }}}{\sigma_{0}^{\prime}}=1
$$

which leads to

$$
\frac{2 A}{c_{v} \lambda^{3}}\left[1-\left(\frac{\lambda z}{2}+1\right) \exp (-\lambda z)\right]=\frac{\left(1+2 K_{o}\right)}{3} \gamma^{\prime} z .
$$

In (16), $\sigma_{0}^{\prime}$ is the effective stress of soil.

Assuming “ $\lambda z$ ” is small, we further expend " $\exp (-\lambda z)$ " with Taylor's expansion, and then use the first three terms, we have the solution of (17) as

$$
z=0
$$

or

$$
z=z_{\text {liq }}=\frac{2}{\lambda} \sqrt{\frac{1}{2}-\frac{\gamma^{\prime}\left(1+2 K_{o}\right) c_{v} \lambda^{2}}{6 A}} .
$$

Note that (18b) is only valid under the condition of $\frac{1}{2}-\frac{\gamma^{\prime}\left(1+2 K_{o}\right) c_{v} \lambda^{2}}{6 A}>0$. It is noted that “ $\frac{1}{2}-\frac{\gamma^{\prime}\left(1+2 K_{o}\right) c_{v} \lambda^{2}}{6 A}<0$ " will provide an unrealistic solution. Under such condition, (18a) will be the solution (i.e., no liquefaction occurs).

Based on (18a) and (18b), a numerical example for the prediction of maximum liquefaction depth $\left(z_{\text {liq }}\right)$ is presented in Figure 5. In the example, the relative water depth $(d / L)$ varies from shallow water $(d / L=0.05)$ to intermediate water $(d / L=0.3)$. As the water depth increases, the maximum wave steepness for inducing soil liquefaction will increase, which will enhance the liquefaction potential. The figure also indicates that the maximum residual liquefaction depth will increase as the wave steepness increases. This finding is also consistent with the result presented in Figure 4 that the residual mechanism is important for the large wave conditions.

\section{Conclusion}

In this study, a new analytical solution for wave-induced residual pore pressure in a porous seabed, which has not been available in the literature, is presented. A comparison of the present model with the existing experimental data ${ }^{[13]}$ indicates that, the present analytical solution overall agrees with the experimental data. Together with previous solution to oscillatory mechanism ${ }^{[10]}$, an amplitude ratio $(\varepsilon)$ is introduced to examine the role of both mechanisms. Numerical results indicate that the residual mechanism is particularly important for large wave loading, while oscillatory mechanism dominates the pore pressure under small wave loading. 


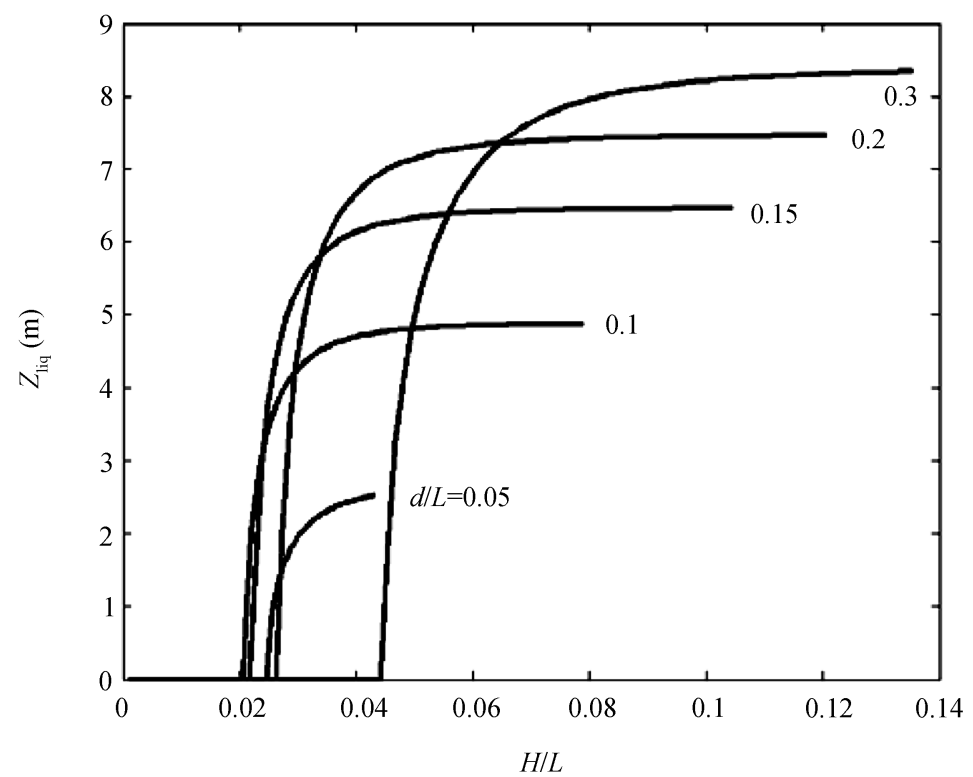

Figure 5 Distribution of maximum liquefaction depth $\left(z_{\mathrm{liq}}\right)$ versus wave steepness $(H / L)$ for various relative water depth $(d / L)$. (Input data: $n_{e}=0.33, \mu=0.33, K=10^{-6} \mathrm{~m} / \mathrm{s}, G=5 \times 10^{6} \mathrm{~N} / \mathrm{m}^{2}, \alpha=0.246, \eta=0.25, c_{v}=0.0022$ and $T=10 \mathrm{~s}$ ).

Based on the new solution, a simplified approximation for the prediction of the maximum liquefaction depth is also proposed for engineering practice. The approximation provides coastal geotechnical engineers a first-hand effective method in the evaluation of the liquefaction potential.

\section{Appendix A: Mathematical derivation of analytical solution}

The boundary value problem for residual pore pressure can be summarized as

$$
\begin{gathered}
\frac{\partial \bar{p}}{\partial t}=c_{v} \frac{\partial^{2} \bar{p}}{\partial z^{2}}+A z e^{-\lambda z}, \\
\bar{p}(0, t)=\bar{p}(z, 0)=0, \text { and } \bar{p}(\infty, t)=0 .
\end{gathered}
$$

Now, we scale the problem as follows:

$$
\varsigma=c_{v} \lambda^{2} t, P=\frac{c_{v} \lambda^{3} \bar{p}}{A} \text { and } y=\lambda z
$$

Then, (A1) and (A2) become

$$
P_{\varsigma}=P_{y y}+y e^{-y} \text { and } P(0, \varsigma)=P(y, 0)=0 .
$$

Take Laplace transformation in $\varsigma$ :

$$
L(P(\varsigma, y))=Q(s, y)=\int_{0}^{\infty} e^{-s \varsigma} P(\varsigma, y) d \varsigma .
$$

Then

$$
s Q=Q_{y y}+\frac{1}{s} y e^{-y} \quad \text { and } \quad Q(s, y)=0, Q(s, \infty)=0 .
$$

The solution of (A6) is

$$
Q(s, y)=\frac{(y s-y-2)}{s(s-1)^{2}} e^{-y}+\frac{2}{s(s-1)^{2}} e^{-\sqrt{s} y} .
$$


We need to invert (A7). Let's split (A7) into two parts:

$$
R(s, y)=\frac{(y s-y-2)}{s(s-1)^{2}} e^{-y} \text { and } V(s, y)=\frac{2}{s(s-1)^{2}} e^{-\sqrt{s} y} .
$$

For $R(s, y)$ the inversion is straight forward: it has a simple pole at $s=0$ and a double pole at $s=1$. $V(s, y)$ has a branch point at $s=0$ and a double pole at $s=1$. This inversion entails a careful integration in the complex s-plane around a "keyhole" contour. The combined inversion gives

$$
P(y, \varsigma)=2\left[1-\left(\frac{y}{2}+1\right)\right] e^{-y}-\frac{1}{\pi} \int_{0}^{\infty} \frac{e^{-r \varsigma}}{r(r+1)^{2}} \sin (\sqrt{r} y) d r .
$$

Now back in dimensional variables,

$$
\bar{p}=\frac{2 A}{c_{v} \lambda^{3}}\left[1-\left(\frac{\lambda z}{2}+1\right) \exp (-\lambda z)-\frac{1}{\pi} \int_{0}^{\infty} \frac{\exp \left(-r c_{v} \lambda^{2} t\right)}{r(r+1)^{2}} \sin (\sqrt{r} \lambda z) d r\right],
$$

which is identical to (13).

The first author is grateful for the support from Institute of Mechanics, Chinese Academy of Sciences under senior visiting scholar scheme and Australian Research Council Linkage-International Award \#LX0455606 (2004-2007).

1 Christian J T, Taylor P K, Yen J K C, et al. Large diameter underwater pipeline for nuclear plant designed against soil liquefaction. In: The 10th Offshore Technology Conference, Houston, 1974, 597—606

2 Dunlap W, Bryant W R, Williams G N, et al. Storm wave effects on deltaic sediments-Results of SEAWAB I and II. Port and Ocean Engineering Under Arctic Conditions (POAC 79). Nerwegian Institute of Technology, 1979, 2: 899—920

3 Miyamoto T, Yoshinaga S, Soga F, et al. Seismic prospecting method applied to the detection of offshore breakwater units setting in the seabed. Coastal Engineering in Japan, 1989, 32: 103-112

4 Nago H, Maeno S, Matsumoto T, et al. Liquefaction and densification of loosely deposited sand bed under wave pressure variation. In: Proceedings of third (1993) International Offshore and Polar Engineering Conference, Singapore, 1993, 578-584

5 Yamamoto T, Koning H L, Sellmejjer H, et al. On the response of a poro-elastic bed to water waves. J Fluid Mech, 1978, 87: 193-206

6 Jeng D -S. Wave-Induced Seabed Response in Front of a Breakwater. PhD Thesis, The University of Western Australia, 1997

7 Seed H B, Rahman M S. Wave-induced pore pressure in relation to ocean floor stability of cohesionless soils. Marine Geotech, 1978, 3: 123-150

8 Sumer B M, Cheng N -S. A radom-walk model for pore pressure accumulation in marine soils. In: Proceedings 9th International Offshore and Polar Engineering Conference. ISOPE-99, Brest, France, 20 May-4 June, 1999, 521-526

9 Biot M A. General theory of three-dimensional consolidation. Journal of Applied Physics, 1941, 12(2): 155-164

10 Jeng D -S, Hsu J R C. Wave-induced soil response in a nearly saturated seabed of finite thickness. Geotech, 1996, 46: $427-440$

11 Sumer B M, Fredsoe J. The Mechanics of Scour in the Marine Environment. New Jersey: World Scientific, Chapter 10, 2002. 445-520

12 McDough W G, Tsai Y T, Liu P L -F, et al. Wave-induced pore water pressure accumulation in marine soils. J Offshore Mech Arctic Engin, A.S.M.E., 1989, 111: 1-11

13 Clukey E C, Kulhawy F H, Liu P L -F. Laboratory and field investigation of wave-sediment interaction. Joseph H. Defrees Hydraulics Laboratory, Report 83-1, School of Civil and Environmental Engineering, Cornell University, Ithaca, N. Y, 1983 